\title{
Four Years Old, One Eye, Pars Planitis and Cataract: Surgical Challenges Give Challenging Surprises
}

\author{
Leonardo García-Rojas Humberto Matiz-Moreno \\ Eduardo Chávez-Mondragón \\ Instituto de Oftalmología Fundación 'Conde de Valenciana' I.A.P., Mexico City, Mexico
}

\section{Key Words}

Pars planitis · Cataract · Posterior synechiae · Iris retractors · Posterior capsulotomy · Membranectomy $\cdot$ Vascular retrolental membrane

\begin{abstract}
Pars planitis (PP) is an intermediate form of uveitis, which, if left untreated, can result in vision loss. Although phacoemulsification with IOL implantation in eyes with PP often results in improved vision, it represents a surgical challenge due to inflammatory sequelae and the possibility of postoperative complications. This case report describes a 4-year-old male who was admitted for poor visual acuity and severe photophobia. The patient previously received vitreo-retinal surgery on his right eye due to an 'unknown disease'. Ophthalmological evaluation revealed NLP, pupillary seclusion and band keratopathy in the right eye; echographic scans revealed funnel retinal detachment. The left eye had a visual acuity of CF at 1 foot, minimal band keratopathy, posterior synechiae and the following echographic findings: abundant vitreous opacities and increased chorioretinal thickness $(1.6 \mathrm{~mm})$. Complete medical and laboratory assessments found no infectious pathologies. The patient was diagnosed with PP. Anti-inflammatory and immunological therapy was given in order to control inflammation. Within four months, the inflammation was controlled. Slow motion phacoemulsification with iris-retractors was performed. During the surgery, an unexpected challenge was identified: an opaque-vascular retrolental membrane. The membrane was cauterized with diathermy. Primary posterior capsulotomy and membranectomy were performed, followed by a limited anterior vitrectomy. An in-the-bag IOL was implanted successfully, with no postoperative complications, and visual acuity improved to 20/200.
\end{abstract}




\section{Introduction}

Pars planitis (PP) is considered as an intermediate form of uveitis, characterized by the aggregation of inflammatory cells and debris on the pars plana (snow banking) or vitreous (snowflakes) in the absence of infection or other associated inflammatory conditions [1]. PP has a relatively common presentation in children and young adults [2]. PP is present in 2.4$15.4 \%$ of patients having uveitis, affecting a greater proportion in the male population $[3,4]$.

Approximately $80 \%$ of cases are bilateral and asymmetric, with symptoms generally limited to one eye [5]. It generally arises as a chronic, insidious and silent cause of permanent vision loss. The most common symptoms are blurred vision and floaters; other, less common symptoms are pain, photophobia and redness of the eyes [4]. PP may generate anterior and posterior segment complications such as cataracts, epiretinal membrane, cystoid macular edema, vascular sheathing, retinal neovascularization, optic disk swelling, vitreous hemorrhage and retinal detachment [4].

Although phacoemulsification with IOL implantation in eyes with PP often results in improved vision, it represents a surgical challenge due to inflammatory sequelae and the possibility of postoperative complications such as reactivation of the PP and progression of cystoid macular edema, submacular fibrosis, epiretinal membrane, significant posterior capsule opacification, IOL deposits, or anterior capsule fibrosis [6].

\section{Case Report}

The patient was a 4-year-old male, admitted for poor visual acuity and severe photophobia. Two years prior, the patient received vitreoretinal surgery on his right eye because of an 'unknown disease' at another hospital. His right eye had a visual acuity of NLP, pupillary seclusion, band keratopathy and a funnel retinal detachment revealed by echographic scans. His left eye (fig. 1) had a visual acuity of CF at 1 foot, minimal band keratopathy, posterior synechiae, cataract and the following echographic findings: abundant vitreous opacities and increased chorioretinal thickness $(1.6 \mathrm{~mm})$. Complete medical and laboratory assessments were performed to determine the presence of infectious and immunological diseases such as toxoplasmosis, toxocariasis, Lyme disease, sarcoidosis, syphilis, tuberculosis, HIV, juvenile rheumatoid arthritis and multiple sclerosis. All of them were negative and no infectious or immunological pathologies were found. On the basis of clinical and echographic findings and those diseases discarded, the patient was diagnosed with PP.

Anti-inflammatory and immunosuppressive therapies such as topical prednisolone and nepafenac as well as oral methotrexate and deflazacort were provided in order to control the inflammation. The patient then returned for multiple clinical and echographical follow-ups. Four months after the initial diagnosis, the inflammation was clinically and echographically controlled in terms of anterior chamber reaction and vitreous opacities, and the medical team, with the consent of the family, decided to perform surgery. Taking his medical history, literature, uveitis and anterior segment services experience into account, a slow-motion phacoemulsification with iris-retractors (fig. 2a) was performed after removing the anterior inflammatory membrane attached to the anterior capsule (fig. 2b). A challenging surprise appeared after the cataract was removed: an opaque-vascular retrolental membrane attached to the posterior capsule (fig. 2c). The membrane vasculature was cauterized with diathermy (fig. 2d) and primary posterior capsulotomy and membranectomy were performed, followed by a limited anterior vitrectomy (fig. 2e). A hydrophobic acrylic one- 
García-Rojas et al.: Four Years Old, One Eye, Pars Planitis and Cataract: Surgical Challenges Give Challenging Surprises

piece IOL was chosen because of its folding ability, a successful in-the-bag implantation and its uveal compatibility (fig. $2 \mathrm{f}$ ). In addition to postoperative antibiotics, anti-inflammatory and immunosuppressive medication was continued (topical prednisolone and nepafenac, and oral methotrexate and deflazacort) and periocular betamethasone was injected three days before surgery to avoid postoperative inflammatory complications. The patient had close monitoring with clinical (fig. 3) and echographical evaluations after surgery. His visual acuity improved to no more than 20/200 three months after the surgery due to the presence of cystoid macular edema. Macular OCT was impossible to get because of the patient's poor cooperation. The patient continues with clinical evaluations, anti-inflammatory and immunosuppressive management (nepafenac, deflazacort and methotrexate) and has required control from ocular hypertension.

\section{Discussion}

PP represents a medical and surgical challenge. Its chronic nature and lack of painful symptoms often result in a late diagnosis, generally not occurring until complications appear. Despite having access to diagnostic and technological tools, there are cases that may be very difficult to study and unpredictable surgical findings may appear. Surgery may produce reactivation of $\mathrm{PP}$ and progression of cystoid macular edema, as well as ocular hypertension due to pupillary block and synechiae, significant posterior capsule opacification, IOL deposits, IOL decentration and anterior capsule fibrosis. The most frequent cause of poor vision recovery that has been reported is cystoid macular edema, submacular fibrosis and epiretinal membrane [6]. Phacoemulsification with IOL implantation in eyes with PP may be safe and has led to good vision outcomes in most cases when precautions are taken, such as pre- and postoperative control of inflammation, meticulous surgery, in-the-bag IOL implantation and vigilant close postoperative care. However, lensectomy plus aphakia may be considered according to clinical findings, inflammation control, surgical resources and surgical findings.

\section{Disclosure Statement}

The authors declare no conflict of interest.

\section{References}

1 Jabs DA, Nussenblatt RB, Rusenbaum JT: Standardization of uveitis nomenclature for reporting clinical data. Results of the first international workshop. Am J Ophthalmol 2005;140:509-516.

Foster CS: Diagnosis and Treatment of Uveitis. Oxford, Elsevier, 2002, pp 844-856.

3 Nikkhah H: Childhood pars planitis; clinical features and outcomes. J Ophtalmic Vis Res 2011;6:249-254.

4 Donaldson MJ: Pars planitis: a 20-year study of incidence, clinical features, and outcomes. Am J Ophtalmol 2007;144:812-817.

5 Romero R, Peralta J, Sendagorta E, Abelairas J: Pars planitis in children: epidemiologic, clinical, and therapeutic characteristics. J Pediatr Ophthalmol Strabismus 2007;44:288-293.

6 Ganesh S, Babu K, Biswas J: Phacoemulsification with intraocular lens implantation in cases of pars planitis. J Cataract Refract Surg 2004;30:2072-2076. 
Presented as a poster on the XXXI National Congress of Ophthalmology, Guadalajara, Jalisco, México, June 2012.

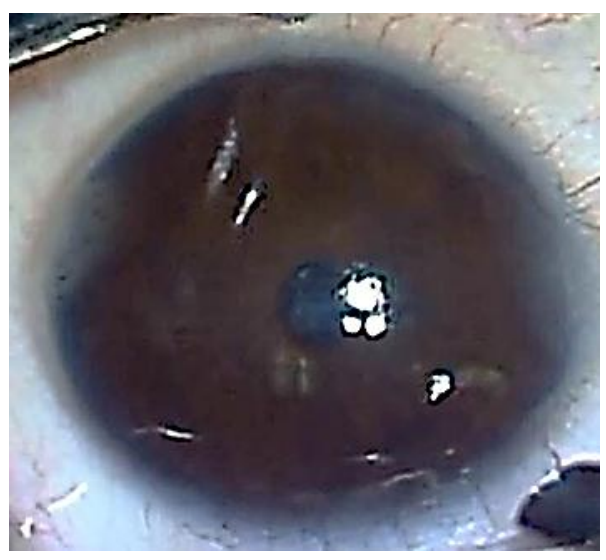

Fig. 1. Left eye before surgery. Band keratopathy, posterior synechiae and cataract.
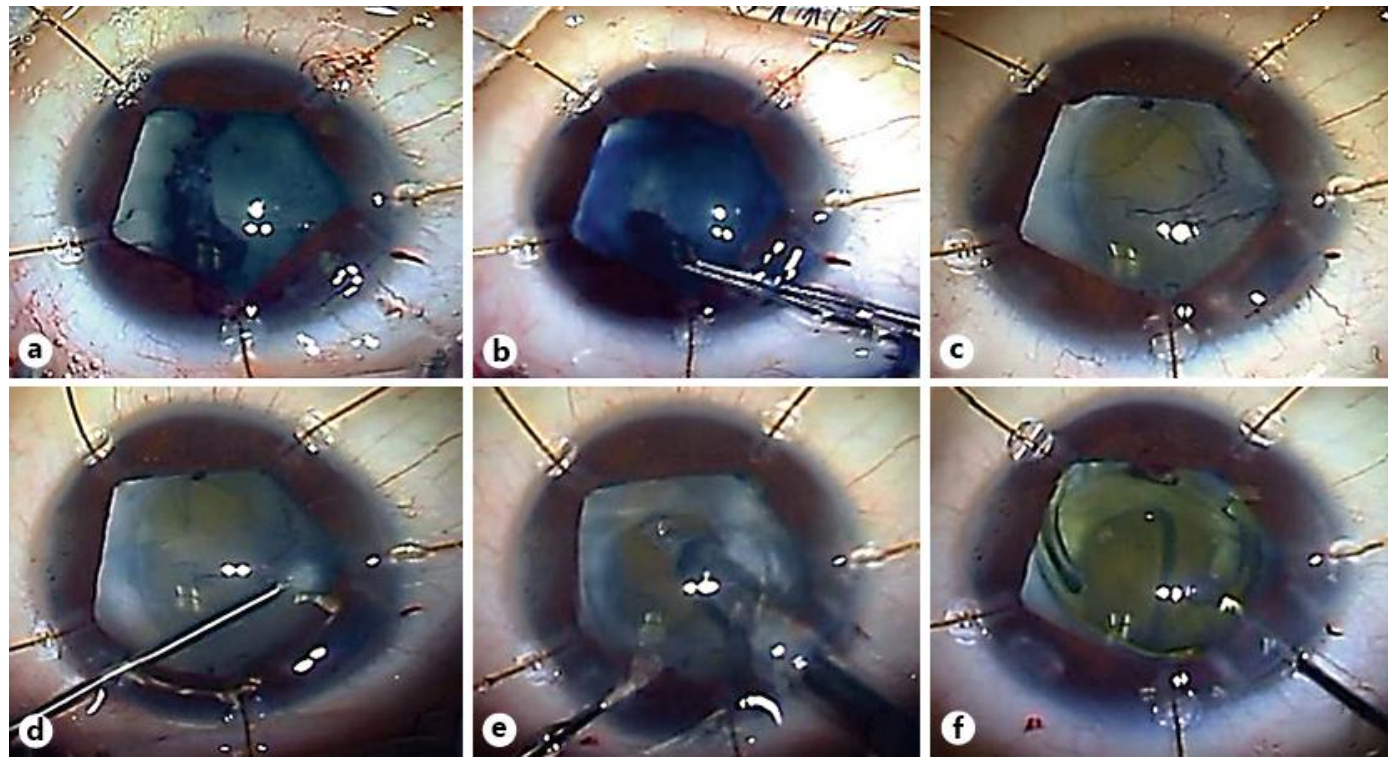

Fig. 2. a Iris retractors. b Anterior inflammatory membrane removal. c Identification of vascular retrolental membrane. d Vasculature cauterization with diathermy. e Posterior membranectomy and automated anterior vitrectomy. $f$ Intraocular lens implantation in the capsular bag. 
García-Rojas et al.: Four Years Old, One Eye, Pars Planitis and Cataract:

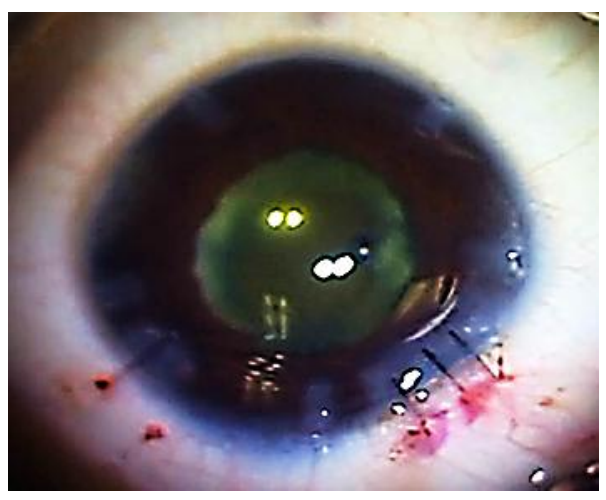

Fig. 3. Left eye after surgery. In-the-bag IOL. 